\title{
吡啶查尔酮衍生物的合成及抑制耐甲氧西林金黄色葡萄球菌活性评价
}

\author{
张 恩 $* a, b$ 王铭铭 ${ }^{a}$ 徐帅民 ${ }^{a}$ 王 上 ${ }^{a}$ 赵 娣 ${ }^{a}$ 白鹏燕 ${ }^{a}$ \\ 崔得运 ${ }^{a}$ 化永刚 ${ }^{a}$ 王亚娜 ${ }^{a}$ 秦上尚*, ${ }^{*}, b \quad$ 刘宏民 ${ }^{a, b}$ \\ $\left({ }^{a}\right.$ 郑州大学药学院 药物研究院 郑州 450001) \\ ( ${ }^{b}$ 新药创制与药物安全性评价河南省协同创新中心 郑州 450001)
}

\begin{abstract}
摘要 合成了一系列 13 个吡啶查尔酮衍生物, 目标化合物的结构通过 ${ }^{1} \mathrm{H}$ NMR、 ${ }^{13} \mathrm{C} N M R$ 和 HRMS 进行了确证. 体 外抗菌活性评价结果显示, 其中的五个化合物对于革兰氏阳性金黄色葡萄球菌(ATCC 29213)表现出良好的抑制活性. 为进一步评估活性, 选取 11 株无重复的耐甲氧西林金黄色葡萄球菌(MRSA)临床株对上述 5 个化合物进行进一步的药 效测评. 数据显示, 其中四个化合物对于 MRSA 表现出较好的抑菌活性, 其中 $(E)-2$-澳- $N$ - $\{4$-[3-(2-吡啶基)丙烯酰基]苯 基\}乙酰胺(5k)对 MRSA 的抑菌效力最为明显, 其最小抑菌浓度(MIC)为 $4 \mu \mathrm{g} / \mathrm{mL}$. 在安全性评价方面, 对化合物 $\mathbf{5 k}$ 进 行红细胞毒性评价, 结果显示化合物 $\mathbf{5 k}$ 即使在 $1000 \mu \mathrm{g} / \mathrm{mL}$ 的浓度下对于红细胞也几乎不存在毒性. 综合上述数据分 析, 吡啶查尔酮 5k 作为新型抗 MRSA 药物具有进一步研究的价值.
\end{abstract}

关键词＼cjkstart吡啶查尔酮；而甲氧西林金黄色葡萄球菌；抗菌活性；红细胞溶血

\section{Synthesis and Antibiotic Activity Study of Pyridine Chalcone Deriva- tives against Methicillin-Resistant Staphylococcus aureus}

\author{
Zhang, En ${ }^{*, a, b} \quad$ Wang, Mingming ${ }^{a} \quad$ Xu, Shuaimin ${ }^{a} \quad$ Wang, Shang ${ }^{a} \quad$ Zhao, Di $^{a}$ \\ Bai, Pengyan $^{a} \quad$ Cui, Deyun $^{a} \quad$ Hua, Yonggang $^{a} \quad$ Wang, Ya'na $^{a}$ \\ Qin, Shangshang*,a,b Liu, Hongmin ${ }^{a, b}$ \\ ( ${ }^{a}$ Institute of Drug Discovery and Development, School of Pharmaceutical Sciences, Zhengzhou University, \\ Zhengzhou 450001) \\ $\left({ }^{b}\right.$ Collaborative Innovation Center of New Drug Research and Safety Evaluation, Henan Province, Zhengzhou 450001)
}

\begin{abstract}
A series of pyridine chalcone derivatives were designed and synthesized. The structures were confirmed by ${ }^{1} \mathrm{H}$ $\mathrm{NMR},{ }^{13} \mathrm{C}$ NMR and HRMS. In vitro biological activity evaluation results showed that five of the compounds exhibited good biological activity against gram positive bacteria staphylococcus aureus (ATCC 29213). Further antibiotic activity of these five compounds against 11 clinical isolated methicillin-resistant staphylococcus aureus (MRSA) were evaluated. The results showed that four of the compounds exhibited good antibacterial activity against MRSA. (E)-2-Bromo- $N$ - $\{4-[3-($ pyridin-2-yl)acryloyl]phenyl $\}$ acetamide (5k) showed the most effective activity (minimum inhibitory concentration, $\mathrm{MIC}=4 \mu \mathrm{g} / \mathrm{mL}$ ). In terms of hemolytic activity evaluation, compound $\mathbf{5 k}$ showed virtually no toxicity even in $1000 \mu \mathrm{g} / \mathrm{mL}$ concentration. To sum up, pyridine chalcone $\mathbf{5} \mathbf{k}$ was potential for further antibiotic study as anti-MRSA agent.
\end{abstract}

Keywords pyridine chalcone; methicillin-resistant Staphylococcus aureus; antibiotic activity; hemolytic activity

耐甲氧西林金黄色葡萄球菌(MRSA)自 1961 年首次 报导以来 ${ }^{[1]}$, 在临床的分离率逐年升高, 并逐渐成为引 起院内感染的重要病原菌 ${ }^{[2,3]}$. MRSA 菌株耐药谱广, 感
染患者病死率高，临床治疗困难. MRSA 多重耐药机制 主要包括两方面: 一是获得了相关的耐药基因; 二是细 菌细胞膜上青霉素结合蛋白(PBP)的改变, 导致 PBP 蛋

\footnotetext{
* Corresponding authors. E-mail: zhangen@zzu.eud.cn; qinshangshang@126.com

Received October 11, 2016; revised December 21, 2016; published online December 29, 2016.

Project supported by the National Natural Science Foundation of China (Nos. U1204206, 81501782) and the Education Department of Henan Province (No. 17A350004).

国家自然科学基金(Nos. U1204206, 81501782)和河南省教育厅(No. 17A350004)资助项目.
} 
白与青霉素类药物结合能力下降 ${ }^{[4]}$. MRSA 的主要传播 方式为皮肤和软组织直接接触传播, 导致病菌扩散 ${ }^{[5]}$. MRSA 院内感染的主要方式是肺炎, 在不合理的治疗方 式下可能最终导致病患死亡. MRSA 自报道发现之日起, 其在美国就以惊人的速度传播, 2003 年美国医院重症监 护室报导发现感染 MRSA 患者的比率高达 $64.4 \%{ }^{[6]}$. 临 床用于治疗 MRSA 的常用药物是糖肽类抗菌药物, 但 是目前在美国已经有 MRSA 对糖肽类药物出现耐药的 报道 ${ }^{[7]}$. 同时对于幼儿和老年患者来说, 糖肽类化合物 的毒副作用也较多 ${ }^{[8]}$. 因此探索新型 MRSA 抑制药物的 研发具有重要的意义.

黄酮和异黄酮是一类重要的天然产物, 广泛存在于 可食用植物中 ${ }^{[9]}$. 查尔酮作为黄酮和异黄酮的核心骨架 单元，广泛存在于天然产物中. 该类化合物具有广泛的 生物学活性, 如抗菌、抗癌、抗真菌、抗炎、抗㾏疾 等 ${ }^{[10 ~ 14]}$. 由于查尔酮的结构中具有 $\alpha, \beta$-不饱和羰基, 可 以与细菌内的亲核性基团(如蛋白质中的颈基)进行共轭 加成而导致细菌死亡 ${ }^{[13]}$. 同时, 由于查尔酮结构单元具 有较好的柔性, 作为底物, 查尔酮就可与多种活性基团 相结合. 研究人员开始关注查尔酮的结构改造, 他们试 图通过结构修饰来开发具有优异抗菌活性的查尔酮类 衍生物 ${ }^{[15 ~ 18]}$. Licochalcone $\mathrm{A}$ 是从甘草里分离得到的查 尔酮化合物, 活性结果表明甘草查尔酮 A 对 MRSA 和临 床分离的万古霉素耐药的屎肠球菌的最小抑菌浓度 (MIC) 值约为 $13 \mu \mathrm{g} / \mathrm{mL}$, 对屎肠球菌的 MIC 值是 $25 \mu \mathrm{g} /$ $\mathrm{mL}^{[17]}$; Cationic Chalcone Antibiotics 78 是新型的脂肪基 团取代的查尔酮衍生物, 其对 MRSA 的 MIC 值为 1 $\mu \mathrm{g} / \mathrm{mL}$, 对大肠埃希菌(ATCC 25922) 也有较好抑菌效果 $(\mathrm{MIC} \text { 为 } 2 \mu \mathrm{g} / \mathrm{mL})^{[17]}$; 二氢查尔酮类化合物 Asebogenin 对金黄色葡萄球菌和 MRSA 的 MIC 值分别为 10 和 4.5 $\mu \mathrm{g} / \mathrm{mL}^{[19]}$ (图 1).

\section{1 结果与讨论}

在前期抗菌活性化合物的合成 ${ }^{[15,16]}$ 和抗肿瘤活性 的查尔酮衍生物合成 ${ }^{[20,21]}$ 的基础上，我们合成了 13 个 吡啶查尔酮衍生物, 力求发现具有良好抗 MRSA 活性 的新型化合物. 合成路线以取代苯乙酮和取代吡啶甲醛 为底物经过羟醛缩合和亲核取代 ${ }^{[12]}$ 两步反应, 得到最 终产物 5a $\sim 5 \mathbf{m}$ (Scheme 1). 合成步骤操作步骤简单, 原 料易得. 化合物最终都通过 ${ }^{1} \mathrm{H} N M R,{ }^{13} \mathrm{C} N M R$ 和 HRMS 进行了结构确证.

首先对于化合物 5a $\sim 5 \mathrm{~m}$ 进行了初步的抗菌活性评 价, 参照美国临床和实验室标准协会(CLSI) M07-A9 标 准, 选取标准菌株革兰氏阳性菌金黄色葡萄球菌(ATCC 29213)和革兰氏阴性菌大肠埃希菌(ATCC 25922), 采用<smiles>C=CC(C)(C)c1cc(/C=C/C(=O)c2ccc(O)cc2)c(OC)cc1O</smiles><smiles></smiles><smiles>Cc1cc(C)cc(-c2ccc(N3CCNCC3)c(C=CC(=O)c3cccc(NCCN(C)C)c3)c2)c1</smiles>

Cationic chalcone antibiotics 78

图 1 具有抗菌活性的部分查尔酮化合物

Figure 1 Some chalcone compounds with antibiotic activity

微量肉汤法测定化合物的 MIC. 利奈唑胺(Linezolid)和 美罗培南(meropenem, MEM)被选为实验组的阳性对照 药物, 实验结果如表 1 所示. 结果表明化合物 $\mathbf{5 a}, \mathbf{5 i}, \mathbf{5 j}$, $5 \mathrm{k}$ 和 $5 \mathrm{~m}$ 对于革兰氏阳性菌金黄色葡萄球菌表现出良好 的抑制活性，对于革兰氏阴性菌大肠埃希菌 (ATCC 25922)于 $64 \mu \mathrm{g} / \mathrm{mL}$ 的浓度下没有抑制作用. 间氨基吡 啶苯乙酮化合物 $5 \mathbf{i}$ 具有较高的抑制金黄色葡萄球菌的 生物活性(MIC 为 $16 \mu \mathrm{g} / \mathrm{mL}$ ), 对氨基吡啶苯乙酩衍生物 $5 \mathbf{a}, 5 \mathbf{j}, 5 \mathbf{k}$ 和 $5 \mathrm{~m}$ 也显示出对于金黄色葡萄球菌(ATCC 29213)良好的抑菌效力(MIC 为 $32 \mu \mathrm{g} / \mathrm{mL}$ ). 然而, 化合 物 5b $\sim 5 h$ 和 51 却显示对于金黄色葡萄球菌没有抑制活 性(其 MIC 值都> $64 \mu \mathrm{g} / \mathrm{mL}$ ). 初步活性结果表明, 该系 列吡啶查尔酮衍生物对革兰氏阳性菌比革兰氏阴性菌 表现出更强的抑制效力.

在初篮的基础上, 又选取了 11 株无重复的耐甲氧 西林金黄色葡萄球菌(MRSA)临床株，对化合物 $\mathbf{5 a}, \mathbf{5 i}$, $5 \mathbf{j}, 5 \mathrm{k}$ 和 $5 \mathrm{~m}$ 进行了进一步的药效学评价, 结果如表 2 所示. 化合物 $5 \mathrm{a}, 5 \mathrm{i}, 5 \mathrm{j}, 5 \mathrm{k}$ 和 $5 \mathrm{~m}$ 都表现出较好的抑菌 (MRSA)活性. 令人兴奋的是, 相对于标准菌株金黄色 葡萄球菌(ATCC 29213), 这 5 个化合物对 MRSA 显示出 更好的抑制活性，这 5 个化合物的 $\mathrm{MIC}_{50}(8 \sim 16 \mu \mathrm{g} / \mathrm{mL})$ 和 $\mathrm{MIC}_{90}(16 \sim 32 \mu \mathrm{g} / \mathrm{mL})$ 都表明了对于 MRSA 的良好抑 菌作用, 尤其是化合物 5k. 化合物 5a, 5i, 5k 和 $5 \mathrm{~m}$ 对于 部分 MRSA 的抗菌的活性要优于临床常用的抗菌药物 左氧氟沙星和红霉素, 但是弱于临床药物利奈唑胺. 而 化合物 $\mathbf{5 k}$ 对于菌株(MRSA) 的抑菌效果 $\left(\mathrm{MIC}_{50}\right.$ 为 8 $\mu \mathrm{g} / \mathrm{mL})$ 与利奈唑胺相当 $\left(\mathrm{MIC}_{50}\right.$ 为 $\left.4 \mu \mathrm{g} / \mathrm{mL}\right)$. 

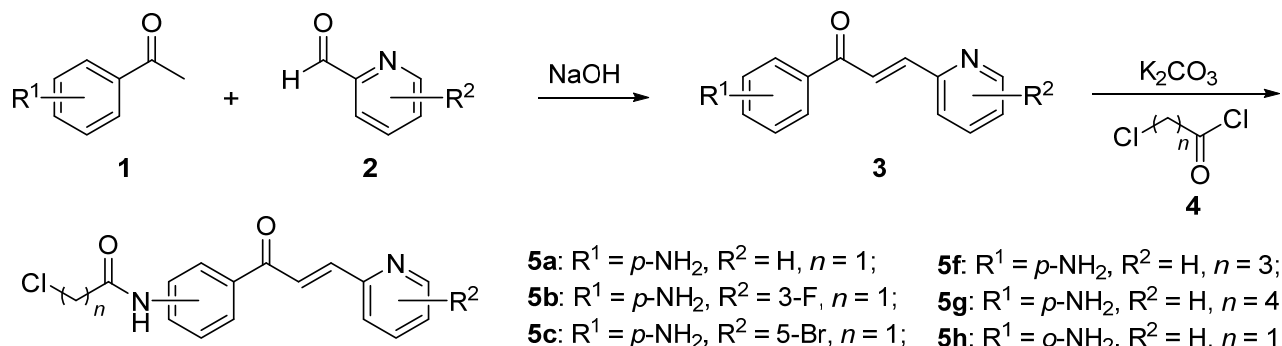

5a: $\mathrm{R}^{1}=p-\mathrm{NH}_{2}, \mathrm{R}^{2}=\mathrm{H}, n=1 ; \quad$ 5f: $\mathrm{R}^{1}=p-\mathrm{NH}_{2}, \mathrm{R}^{2}=\mathrm{H}, n=3$;

5b: $\mathrm{R}^{1}=p-\mathrm{NH}_{2}, \mathrm{R}^{2}=3-\mathrm{F}, n=1 ; \quad \mathbf{5 g}: \mathrm{R}^{1}=p-\mathrm{NH}_{2}, \mathrm{R}^{2}=\mathrm{H}, n=4$

5c: $\mathrm{R}^{1}=p-\mathrm{NH}_{2}, \mathrm{R}^{2}=5-\mathrm{Br}, n=1 ; \quad 5 \mathbf{h}: \mathrm{R}^{1}=o-\mathrm{NH}_{2}, \mathrm{R}^{2}=\mathrm{H}, n=1$

5d: $\mathrm{R}^{1}=p-\mathrm{NH}_{2}, \mathrm{R}^{2}=6-\mathrm{Br}, n=1 ; \quad 5 \mathrm{i}: \mathrm{R}^{1}=m-\mathrm{NH}_{2}, \mathrm{R}^{2}=\mathrm{H}, n=1$

5e: $\mathrm{R}^{1}=p-\mathrm{NH}_{2}, \mathrm{R}^{2}=\mathrm{H}, n=2$<smiles>CC(=O)Nc1ccc(C(=O)/C=C/c2ccccn2)cc1</smiles><smiles>O=C(CBr)Nc1ccc(C(=O)/C=C/c2ccccn2)cc1</smiles><smiles>O=C(CCl)Nc1ccc(C(=O)/C=C/c2cccnc2)cc1</smiles><smiles>O=C(CCl)Nc1ccc(C(=O)/C=C/c2ccncc2)cc1</smiles>

图式 1 化合物 5a $\sim 5 \mathrm{~m}$ 的合成路线

Scheme 1 Synthetic route of compounds $5 \mathrm{a} \sim 5 \mathrm{~m}$

表 1 化合物 $\mathbf{5 a} \sim \mathbf{5 m}$ 的最小抑菌浓度 $(\mu \mathrm{g} / \mathrm{mL})^{a}$

Table 1 MIC data $(\mu \mathrm{g} / \mathrm{mL})$ of compounds $\mathbf{5 a} \sim \mathbf{5 m}$

\begin{tabular}{|c|c|c|c|c|c|c|c|c|}
\hline \multirow{2}{*}{ 化合物 } & \multicolumn{2}{|c|}{ 临床敏感菌株 } & \multirow{2}{*}{ 化合物 } & \multicolumn{2}{|c|}{ 临床敏感菌株 } & \multirow{2}{*}{ 化合物 } & \multicolumn{2}{|c|}{ 临床敏感菌株 } \\
\hline & ATCC 29213 & ATCC 25922 & & ATCC 29213 & ATCC 25922 & & ATCC 29213 & ATCC 25922 \\
\hline $5 a$ & 32 & $>64$ & $5 f$ & $>64$ & $>64$ & $5 \mathbf{k}$ & 32 & $>64$ \\
\hline $5 \mathbf{b}$ & $>64$ & $>64$ & $5 g$ & $>64$ & $>64$ & 51 & $>64$ & $>64$ \\
\hline $5 c$ & $>64$ & $>64$ & $5 \mathrm{~h}$ & $>64$ & $>64$ & $5 \mathrm{~m}$ & 32 & $>64$ \\
\hline $5 d$ & $>64$ & $>64$ & $5 \mathbf{i}$ & 16 & $>64$ & 利奈唑胺 ${ }^{b}$ & 2 & $-^{c}$ \\
\hline $5 e$ & $>64$ & $>64$ & $5 \mathbf{j}$ & 32 & $>64$ & 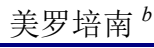 & $-^{c}$ & $<0.125$ \\
\hline
\end{tabular}

a 能抑制培养基内病原菌生长的最低药物浓度; ${ }^{b}$ 阳性对照药物; ${ }^{c}$ 未测.

表 2 化合物 $\mathbf{5 a}, \mathbf{5 i}, \mathbf{5 j}, \mathbf{5 k}$ 和 $\mathbf{5 m}$ 最小抑菌浓度(MIC)的测定和 $\mathrm{MIC}_{50}, \mathrm{MIC}_{90}$ 结果 $(\mu \mathrm{g} / \mathrm{mL})$

Table 2 MIC, $\mathrm{MIC}_{50}, \mathrm{MIC}_{90}$ data $(\mu \mathrm{g} / \mathrm{mL})$ of compounds $\mathbf{5 a}, \mathbf{5 i}, \mathbf{5 j}, \mathbf{5 k}$ and $\mathbf{5 m}$

\begin{tabular}{|c|c|c|c|c|c|c|c|c|c|c|c|c|c|}
\hline \multirow{2}{*}{ 化合物 } & \multicolumn{11}{|c|}{ MIC (MRSA) } & \multirow{2}{*}{$\mathrm{MIC}_{50}{ }^{a}$} & \multirow{2}{*}{$\mathrm{MIC}_{90}{ }^{b}$} \\
\hline & $1^{c}$ & $2^{c}$ & $3^{c}$ & $4^{c}$ & $5^{c}$ & $6^{c}$ & $7^{c}$ & $8^{c}$ & $9^{c}$ & $10^{c}$ & $11^{c}$ & & \\
\hline $5 a$ & 16 & 16 & 16 & 16 & 16 & 16 & 16 & 16 & 16 & 16 & 32 & 16 & 16 \\
\hline $5 \mathbf{i}$ & 16 & 16 & 16 & 8 & 64 & 16 & 32 & 32 & 16 & 16 & 32 & 16 & 32 \\
\hline $5 \mathbf{j}$ & $>64$ & $>64$ & $>64$ & $>64$ & $>64$ & $>64$ & $>64$ & $>64$ & $>64$ & $>64$ & $>64$ & $>64$ & $>64$ \\
\hline $5 \mathbf{k}$ & 8 & 8 & 8 & 4 & 16 & 8 & 16 & 8 & 8 & 8 & 16 & 8 & 16 \\
\hline $5 \mathrm{~m}$ & 16 & 16 & 16 & 8 & 32 & 16 & 16 & 8 & 8 & 16 & 16 & 16 & 16 \\
\hline 左氧氟沙星 ${ }^{d}$ & $>64$ & $>64$ & $>64$ & $>64$ & $>64$ & $>64$ & $>64$ & $>64$ & $>64$ & $>64$ & 0.25 & $>64$ & $>64$ \\
\hline 红霉素 ${ }^{d}$ & 4 & 2 & 0.5 & $>64$ & $>64$ & $>64$ & $>64$ & $>64$ & $>64$ & $>64$ & 0.25 & $>64$ & $>64$ \\
\hline 利奈唑胺 ${ }^{d}$ & 4 & 4 & 1 & 2 & 4 & 4 & 4 & 4 & 4 & 4 & 2 & 4 & 4 \\
\hline
\end{tabular}

a 在一批实验中能抑制 $50 \%$ 数目的菌株生长的 $\mathrm{MIC} ;{ }^{b}$ 在一批实验中能抑制 $90 \%$ 数目的菌株生长的 $\mathrm{MIC} ;{ }^{c}$ 耐甲氧西林金黄色葡萄球菌临床分离株的编号; 阳性对照药物。

分析不同的化合物结构对金黄色葡萄球菌抑制活 性的影响，化合物 $5 \mathbf{a}, 5 \mathbf{j}, 5 \mathbf{k}, 5 \mathbf{i}$ 和 $5 \mathrm{~m}$ 都是由对氨基吡 啶苯乙酮为底物衍生得到，相对邻位氨基和间位氨基取 代的吡定苯乙酮，对位氨基吡啶苯乙酩衍生物具有更好 的抑制 MRSA 的活性. 从化合物 $\mathbf{5 a}$ 对金黄色葡萄球菌 的抑制活性优于化合物 $\mathbf{5 b} \sim \mathbf{5 g}$ 中可以看出, 增加碳链 长度和利用卤素原子取代的邻位吡啶甲醛对抗菌活性 并没有任何改善. 通过对化合物 5a, 5l 和 $5 \mathrm{~m}$ 的结构活 性分析比较可以看出, 邻位吡啶甲醛衍生物较对位吡啶 甲醛和间位吡啶甲醛衍生物具有更好的抑制金黄色葡
萄球菌生物活性的潜力. 对作用于 MRSA 的活性结果 分析，临床常用抗菌药物左氧氟沙星仅对编号为 11 的 菌株有效，红霉素仅对编号为 1, 2, 3 和 11 号菌株有效; 化合物 5a 和 $5 \mathbf{i}$ 对于 MRSA 的抗菌结果表明, 芳环上氨 基取代的位置对于其 MIC 几乎没有影响; 由化合物 $\mathbf{5 j}$, $\mathbf{5 k}, \mathbf{5 m}$ 对于 MRSA 的抑菌结果分析可得, 当末端为甲 基时，化合物 $\mathbf{5 j}$ 不存在抵抗 MRSA 的效力, 然而当末端 为亚甲基溴存在的情况下，化合物 $5 \mathbf{k}$ 很大程度的提高 了抵抗 MRSA 的抗菌效力, 并且 MIC 值最小可以达到 4 $\mu \mathrm{g} / \mathrm{mL}$. 
为了初步测试该类化合物的毒性, 我们对化合物 $\mathbf{5 k}$ 用红细胞溶血法进行体外毒性评价, 溶血活性结果 如图 2 所示. 当化合物 $\mathbf{5 k}$ 的浓度在 $2 \mu \mathrm{g} / \mathrm{mL}$ 到 1000 $\mu \mathrm{g} / \mathrm{mL}$ 之间, 其对红细胞毒性很小. 即使化合物 $\mathbf{5 k}$ 在远 大于其 MIC 的浓度 $1000 \mu \mathrm{g} / \mathrm{mL}$ 时, 依然只能导致大约 $1 \%$ 的红细胞发生溶血. 实验结果证实, 化合物 $\mathbf{5 k}$ 对血 红细胞毒性很低, 表明化合物 $\mathbf{5 k}$ 有一个广泛的安全范 围.

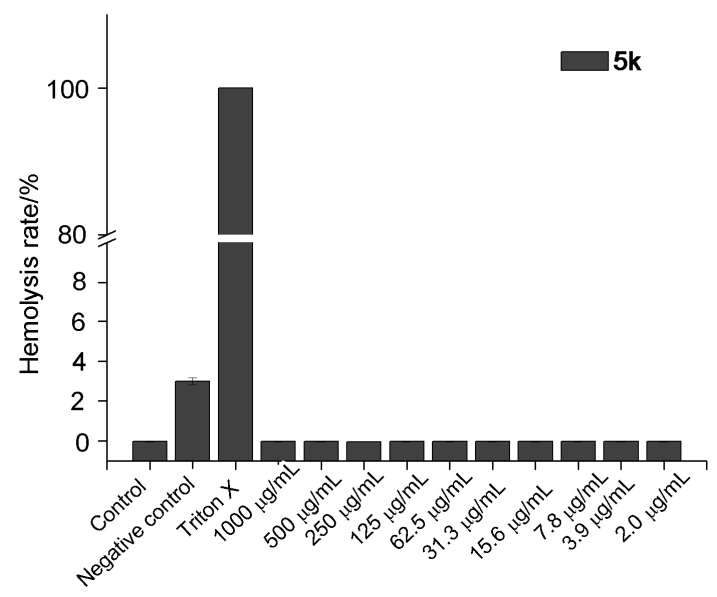

图 2 化合物 $\mathbf{5 k}$ 的红细胞毒性结果

Figure 2 Toxicity against RBCs of $\mathbf{5 k}$

\section{2 结论}

合成了一系列的吡啶查尔酮衍生物, 并对其体外生 物活性进行了测定, 发现部分化合物具有抑制临床耐药 菌株 MRSA 的药理学活性. 活性实验结果表明化合物 $5 a, 5 i, 5 j, 5 k$ 和 $5 \mathrm{~m}$ 对革兰氏阳性细菌具有较好的抑制 活性, 该系列化合物在 $64 \mu \mathrm{g} / \mathrm{mL}$ 浓度下对于革兰氏阴 性细菌不存在抑菌活性. 此外, 化合物 $5 \mathbf{a}, 5 \mathbf{5 i}, 5 \mathbf{j}, 5 \mathbf{k}$ 和 $5 \mathrm{~m}$ 在对 11 株临床分离的耐药菌株 MRSA 的抑制活性 数据显示, 它们中的大多数表现出具有比阳性对照药物 (左氧氟沙星和红霉素)更好的抗菌活性. 其中, 化合物 $\mathbf{5 k}$ 对临床分离的 11 株 MRSA 的 $\mathrm{MIC}_{50}$ 可以达到 8 $\mu \mathrm{g} / \mathrm{mL}$, 比阳性对照利奈唑胺稍差. 化合物 $\mathbf{5 k}$ 的红细胞 毒性数据显示, 该化合物即使在浓度为 $1000 \mu \mathrm{g} / \mathrm{mL}$ 的 条件下, 也几乎不存在红细胞毒性, 这就表明其安全窗 口远大于其最低抑菌浓度(MIC), 这使得其具备作为抗 MRSA 抑制剂进一步研究的潜力.

\section{3 实验部分}

\section{1 仪器与试剂}

核磁共振仪(Bruker DPX400), 质谱仪(Waters-Micromass 公司 Q-Tof 质谱仪), 旋转蒸发仪(东京理化), 实验所用柱层析硅胶及薄层层析硅胶板购自青岛海洋
化工厂. 所用试剂均为国产或进口分析纯, 无水溶剂用 常规方法干燥处理.

\section{2 实验方法}

\subsection{1 (E)-4-氨基-(2-吡啶基)苯丙烯酮(3a)的合成}

取氢氧化钠 $(370 \mathrm{mg}, 9.25 \mathrm{mmol})$ 于单口圆底烧瓶 $(250 \mathrm{~mL})$ 中, 加入水 $(50 \mathrm{~mL})$, 室温摚拌下溶解; 然后将 对氨基苯乙酮(1a) (1.00 g, $7.40 \mathrm{mmol}$ )和 2-吡啶甲醛(2a) $(722 \mu \mathrm{L}, 7.58 \mathrm{mmol})$ 加入 $100 \mathrm{~mL}$ 锥形瓶内, 加入乙醇 $(50 \mathrm{~mL})$ 超声至该体系基本澄清, 然后将该澄清溶液恒 压滴加 $(1$ 滴/ $\mathrm{s})$ 到上述氢氧化钠溶液的单口瓶内, 滴加完 毕后室温下继续反应. 约 $6 \mathrm{~h}$ 后, 薄层色谱(TLC)检测 $[V($ 石油醚 $): V($ 乙酸乙酯 $)=1: 1]$ 显示反应完全. 停止 反应, 将反应体系倒入冰水 $(50 \mathrm{~mL})$ 中, 析出大量黄色固 体, 抽滤, 滤饼水洗至中性, 真空干燥, 得到亮黄色固 体化合物 3a (1.48 g), 收率为 $89 \% .{ }^{1} \mathrm{H}$ NMR $(400 \mathrm{MHz}$, DMSO- $\left.d_{6}\right) \delta: 8.68(\mathrm{~d}, J=4.7 \mathrm{~Hz}, 1 \mathrm{H}), 8.13(\mathrm{~d}, J=15.4$ $\mathrm{Hz}, 1 \mathrm{H}), 7.95 \sim 7.84(\mathrm{~m}, 4 \mathrm{H}), 7.63(\mathrm{~d}, J=15.4 \mathrm{~Hz}, 1 \mathrm{H})$, $7.45 \sim 7.38(\mathrm{~m}, 1 \mathrm{H}), 6.66(\mathrm{~d}, J=8.6 \mathrm{~Hz}, 2 \mathrm{H}), 6.25(\mathrm{~s}, 2 \mathrm{H})$; ${ }^{13} \mathrm{C}$ NMR (101 MHz, DMSO- $\left.d_{6}\right) \quad \delta$ : 185.79, 154.11, $153.32,149.87,140.59,137.07,131.13,125.55,125.05$, $124.38,124.29,112.82$.

\subsection{2 (E)-3-氨基-(2-吡啶基)苯丙烯酮 $(3 \mathbf{b})$ 的合成}

操作同 3a 的合成, 产物为黄色固体, 收率为 $64 \%$. ${ }^{1} \mathrm{H}$ NMR $\left(400 \mathrm{MHz}, \mathrm{CDCl}_{3}\right) \delta: 8.68(\mathrm{~d}, J=4.0 \mathrm{~Hz}, 1 \mathrm{H})$, $8.06(\mathrm{~d}, J=15.3 \mathrm{~Hz}, 1 \mathrm{H}), 7.78 \sim 7.70(\mathrm{~m}, 2 \mathrm{H}), 7.47(\mathrm{dd}$, $J=7.7,4.1 \mathrm{~Hz}, 2 \mathrm{H}), 7.40 \sim 7.36(\mathrm{~m}, 1 \mathrm{H}), 7.29$ (t, $J=3.4$ $\mathrm{Hz}, 1 \mathrm{H}), 7.25$ (s, 1H), 6.90 (dd, $J=7.9,2.3 \mathrm{~Hz}, 1 \mathrm{H}), 3.87$ (s, 2H); ${ }^{13} \mathrm{C}$ NMR $\left(101 \mathrm{MHz}, \mathrm{CDCl}_{3}\right) \delta: 190.58,153.29$, $150.13,146.86,142.45,138.90,136.86,129.49,125.81$, $125.32,124.33,119.68,119.13,114.51$.

\subsection{3 (E)-2-氨基-(2-吡啶基)苯丙烯酮 $(3 \mathrm{c})$ 的合成}

操作同 3a 的合成, 产物为黄色固体, 收率为 $68 \%$. ${ }^{1} \mathrm{H}$ NMR $\left(400 \mathrm{MHz}\right.$, DMSO- $\left.d_{6}\right) \delta: 8.68(\mathrm{~d}, J=4.7 \mathrm{~Hz}$, $1 \mathrm{H}), 8.20(\mathrm{~d}, J=15.3 \mathrm{~Hz}, 1 \mathrm{H}), 7.97(\mathrm{dd}, J=8.2,1.0 \mathrm{~Hz}$, 1H), 7.89 (d, $J=3.5 \mathrm{~Hz}, 2 \mathrm{H}), 7.63(\mathrm{~d}, J=15.3 \mathrm{~Hz}, 1 \mathrm{H})$, $7.51 \sim 7.36(\mathrm{~m}, 3 \mathrm{H}), 7.36 \sim 7.24(\mathrm{~m}, 1 \mathrm{H}), 6.83(\mathrm{~d}, J=7.7$ $\mathrm{Hz}, 1 \mathrm{H}), 6.64 \sim 6.55(\mathrm{~m}, 1 \mathrm{H}) ;{ }^{13} \mathrm{C}$ NMR $(101 \mathrm{MHz}$, DMSO- $\left.d_{6}\right) \delta: 190.40,153.18,152.12,149.90,140.99$, $137.09,134.51,131.19,126.52,124.42,124.38,117.22$, $116.97,114.56$.

\subsection{4 (E)-3-氨基-(3-吡啶基)苯丙烯酮 (3d) 的合成}

操作同 3a 的合成, 产物为黄色固体, 收率为 $72 \%$. ${ }^{1} \mathrm{H}$ NMR (400 MHz, DMSO- $\left.d_{6}\right) \delta: 8.99(\mathrm{~d}, J=1.8 \mathrm{~Hz}$, $1 \mathrm{H}), 8.59(\mathrm{dd}, J=4.7,1.4 \mathrm{~Hz}, 1 \mathrm{H}), 8.32(\mathrm{~d}, J=8.0 \mathrm{~Hz}$, 
1H), 7.99 (dd, $J=23.1,12.0 \mathrm{~Hz}, 3 \mathrm{H}), 7.65$ (d, $J=15.6 \mathrm{~Hz}$, $1 \mathrm{H}), 7.48$ (dd, $J=7.8,4.9 \mathrm{~Hz}, 1 \mathrm{H}), 6.65$ (d, $J=8.4 \mathrm{~Hz}$, 2H), $6.22(\mathrm{~s}, 2 \mathrm{H}) ;{ }^{13} \mathrm{C}$ NMR (101 MHz, DMSO-d $\left.d_{6}\right) \delta$ : $130.95,125.05,124.35,123.82,112.72$.

\subsection{5 (E)-3-氨基-(4-吡啶基)苯丙烯酮 $(3 e)$ 的合成}

操作同 $3 \mathrm{a}$ 的合成, 产物为黄色固体, 收率为 $62 \%$. ${ }^{1} \mathrm{H}$ NMR (400 MHz, DMSO- $\left.d_{6}\right) \delta: 8.65$ (d, $J=5.4 \mathrm{~Hz}$, 2H), 8.09 (d, $J=15.6 \mathrm{~Hz}, 1 \mathrm{H}), 7.96$ (d, $J=8.7 \mathrm{~Hz}, 2 \mathrm{H})$, 7.80 (d, $J=5.9 \mathrm{~Hz}, 2 \mathrm{H}), 7.56$ (d, $J=15.6 \mathrm{~Hz}, 1 \mathrm{H}), 6.65$ (d, $J=8.7 \mathrm{~Hz}, 2 \mathrm{H}), 6.26(\mathrm{~s}, 2 \mathrm{H}) ;{ }^{13} \mathrm{C} \mathrm{NMR}(101 \mathrm{MHz}$, DMSO- $\left.d_{6}\right) \delta: 185.46,154.24,150.23,142.31,138.47$, 131.40, 126.84, 124.87, 122.29, 112.75 .

$3.2 .6(E)-4$-氨基-(3-氟-2-吡啶基)苯丙烯酮 $(\mathbf{3 f})$ 的合 成

操作同 $3 \mathrm{a}$ 的合成, 产物为黄色固体, 收率为 $58 \%$. ${ }^{1} \mathrm{H}$ NMR (400 MHz, DMSO- $\left.d_{6}\right) \delta: 8.55(\mathrm{~d}, J=4.5 \mathrm{~Hz}$, $1 \mathrm{H}), 8.16(\mathrm{~d}, J=15.3 \mathrm{~Hz}, 1 \mathrm{H}), 7.90 \sim 7.71(\mathrm{~m}, 4 \mathrm{H}), 7.55$ (dt, $J=8.6,4.4 \mathrm{~Hz}, 1 \mathrm{H}), 6.66$ (d, $J=8.7 \mathrm{~Hz}, 2 \mathrm{H}), 6.29$ (s, $2 \mathrm{H}) ;{ }^{13} \mathrm{C}$ NMR (101 MHz, DMSO- $\left.d_{6}\right) \delta: 185.31,157.85$, $154.29,146.12$, 141.09, 131.64, 131.16, 126.91, 126.51, $124.81,124.34,112.90$.

3.2.7 (E)-4-氨基-(5-溴-2-吡啶基)苯丙烯酮 $(3 \mathrm{~g})$ 的合 成

操作同 $3 \mathrm{a}$ 的合成, 产物为黄色固体, 收率为 $78 \%$. ${ }^{1} \mathrm{H}$ NMR (400 MHz, $\left.\mathrm{CDCl}_{3}\right) \delta: 8.71(\mathrm{~d}, J=2.3 \mathrm{~Hz}, 1 \mathrm{H})$, 8.10 (d, $J=15.2 \mathrm{~Hz}, 1 \mathrm{H}), 7.99$ (d, $J=8.7 \mathrm{~Hz}, 2 \mathrm{H}), 7.85$ (dd, $J=8.3,2.4 \mathrm{~Hz}, 1 \mathrm{H}), 7.67$ (d, $J=15.2 \mathrm{~Hz}, 1 \mathrm{H}), 7.34$ (d, $J=8.3 \mathrm{~Hz}, 1 \mathrm{H}), 6.70$ (d, $J=8.7 \mathrm{~Hz}, 2 \mathrm{H}), 4.22$ (s, 2H); ${ }^{13} \mathrm{C} \mathrm{NMR}\left(101 \mathrm{MHz}, \mathrm{CDCl}_{3}\right) \delta: 187.74,152.12,151.45$, $151.22,139.81,139.36,131.42,128.19,126.33,126.04$, 121.14, 113.94 .

\subsection{8 (E)-4-氨基-(6-溴-2-吡啶基)苯丙烯酮 $(3 \mathbf{h})$ 的合 成}

操作同 $3 \mathrm{a}$ 的合成, 产物为黄色固体, 收率为 $74 \%$. ${ }^{1} \mathrm{H}$ NMR (400 MHz, $\left.\mathrm{CDCl}_{3}\right) \delta: 8.11(\mathrm{~d}, J=15.1 \mathrm{~Hz}, 1 \mathrm{H})$, $7.99(\mathrm{~d}, J=8.6 \mathrm{~Hz}, 2 \mathrm{H}), 7.67 \sim 7.52(\mathrm{~m}, 2 \mathrm{H}), 7.45(\mathrm{~d}, J=$ $7.8 \mathrm{~Hz}, 1 \mathrm{H}), 7.37$ (d, $J=7.4 \mathrm{~Hz}, 1 \mathrm{H}), 6.70$ (d, $J=8.6 \mathrm{~Hz}$, 2H), $4.24(\mathrm{~s}, 2 \mathrm{H}) ;{ }^{13} \mathrm{C}$ NMR (101 MHz, $\left.\mathrm{CDCl}_{3}\right) \delta: 187.65$, $154.91,151.49,142.58,139.24,139.02,131.50,128.41$, $128.11,127.15,123.90,113.94$.

\section{2 .9 (E)-2-氯- $N$ - $\{4$-[3-(2-吡啶基)丙烯酰基]苯基 $\} 乙$} 酰胺 $(\mathbf{5 a})$ 的合成

取上述化合物 3a (100 mg, $0.446 \mathrm{mmol})$ 和碳酸钾 (74 $\mathrm{mg}, 0.535 \mathrm{mmol})$ 于单口圆底烧瓶 $(5 \mathrm{~mL})$, 然后加入丙酮 $(1.35 \mathrm{~mL})$, 室温下搅拌; 然后用 $1 \mathrm{~mL}$ 注射器向反应体系
注入化合物 $4(40.3 \mu \mathrm{L}, 0.535 \mathrm{mmol})$, 体系由原来的橙色 浑浊变为黄色浑浊. 约 $0.5 \mathrm{~h}$ 后, TLC [ $V$ (石油醚) : $V($ 乙 酸乙酯 $)=1: 1]$ 检测, 显示反应完全, 加入 $3 \mathrm{~mL}$ 冰水淬 灭反应, 搅拌 $10 \mathrm{~min}$ 后, 将体系抽滤, 滤饼冰水洗至中 性, 真空干燥得到化合物 $\mathbf{5 a}(119 \mathrm{mg}), \mathbf{5 a}$ 为浅棕色固体, 收率为 79\%. m.p. 137 138 ${ }^{\circ} \mathrm{C}$; ${ }^{1} \mathrm{H}$ NMR (400 MHz, $\left.\mathrm{CDCl}_{3}\right) \delta: 8.70(\mathrm{~d}, J=3.9 \mathrm{~Hz}, 1 \mathrm{H}), 8.51(\mathrm{~s}, 1 \mathrm{H}), 8.18 \sim$ $8.08(\mathrm{~m}, 3 \mathrm{H}), 7.82 \sim 7.68(\mathrm{~m}, 4 \mathrm{H}), 7.48(\mathrm{~d}, J=7.7 \mathrm{~Hz}$, 1H), 7.31 (ddd, $J=7.6,4.8,0.9 \mathrm{~Hz}, 1 \mathrm{H}), 4.23$ (s, 2H); ${ }^{13} \mathrm{C}$ NMR (101 MHz, $\left.\mathrm{CDCl}_{3}\right) \delta: 188.86,164.07,153.11$, $150.13,142.66,141.01,136.98,134.34,130.22,125.52$, 125.23, 124.49, 119.37, 42.89. HRMS (ESI) calcd for $\mathrm{C}_{16} \mathrm{H}_{14} \mathrm{ClN}_{2} \mathrm{O}_{2}[\mathrm{M}+\mathrm{H}]^{+}:$301.0744, found 301.0744.

3.2.10 (E)-2-氯- $N$ - $\{4$-[3-(3-氟吡啶-2-基)丙烯酰基] 苯基 $\}$ 乙酰胺 $(\mathbf{5 b})$ 的合成

操作同 $5 \mathbf{a}$ 的合成, 产物为淡黄色固体, 收率为 78\%. m.p. 157 $158{ }^{\circ} \mathrm{C}$; ${ }^{1} \mathrm{H}$ NMR (400 MHz, $\left.\mathrm{CDCl}_{3}\right) \delta$ : 8.43 (d, $J=4.4 \mathrm{~Hz}, 1 \mathrm{H}), 8.39$ (s, 1H), 8.10 (dd, $J=24.8$, $12.0 \mathrm{~Hz}, 3 \mathrm{H}), 7.98$ (dd, $J=15.3,1.1 \mathrm{~Hz}, 1 \mathrm{H}), 7.66$ (d, $J=$ $8.7 \mathrm{~Hz}, 2 \mathrm{H}), 7.48 \sim 7.33(\mathrm{~m}, 1 \mathrm{H}), 7.28(\mathrm{dt}, J=8.5,4.3 \mathrm{~Hz}$, $1 \mathrm{H}), 4.16(\mathrm{~s}, 2 \mathrm{H}) ;{ }^{13} \mathrm{C} \mathrm{NMR}\left(101 \mathrm{MHz}, \mathrm{CDCl}_{3}\right) \delta: 188.57$, $164.04,158.58,145.75,141.89141 .04,134.69,134.30$, $130.27,126.46,125.95,123.93,119.39,42.89$. HRMS (ESI) calcd for $\mathrm{C}_{16} \mathrm{H}_{13} \mathrm{ClFN}_{2} \mathrm{O}_{2}[\mathrm{M}+\mathrm{H}]^{+}: 319.0650$, found 319.0648 .

$3.2 .11(E)-\{4-[3-(5$ - 溴-2-吡啶基)丙烯酰基]-2-氯苯 基 $\}$ 乙酰胺 $(\mathbf{5 c})$ 的合成

操作同 $5 \mathbf{a}$ 的合成, 产物为淡黄色固体, 收率为 75\%. m.p. 226 $227{ }^{\circ} \mathrm{C} ;{ }^{1} \mathrm{H}$ NMR (400 MHz, DMSO- $d_{6}$ ) $\delta: 10.70(\mathrm{~s}, 1 \mathrm{H}), 8.81(\mathrm{~d}, J=2.2 \mathrm{~Hz}, 1 \mathrm{H}), 8.22 \sim 8.10(\mathrm{~m}$, 4H), 7.92 (d, $J=8.4 \mathrm{~Hz}, 1 \mathrm{H}), 7.80$ (d, $J=8.7 \mathrm{~Hz}, 2 \mathrm{H}), 7.69$ (d, $J=15.4 \mathrm{~Hz}, 1 \mathrm{H}), 4.33(\mathrm{~s}, 2 \mathrm{H}) ;{ }^{13} \mathrm{C} \mathrm{NMR}(101 \mathrm{MHz}$, DMSO- $\left.d_{6}\right) \delta: 187.62,165.24,151.71,150.72,143.10$, $141.25,139.74,132.40,130.01,126.28,125.69,121.21$, 118.85, 43.59. HRMS (ESI) calcd for $\mathrm{C}_{16} \mathrm{H}_{13} \mathrm{BrClN}_{2} \mathrm{O}_{2}$ $[\mathrm{M}+\mathrm{H}]^{+}: 378.9849$, found 378.9850 .

\section{$3.2 .12(E)-\{4-[3-(6$ - 溴-2-吡啶基)丙烯酰基]-2-氯苯} 基 $\}$ 乙酰胺 $(\mathbf{5 d})$ 的合成

操作同 $\mathbf{5 a}$ 的合成, 产物为淡黄色固体, 收率为 73\%. m.p. 225 226 ${ }^{\circ} \mathrm{C}$; ${ }^{1} \mathrm{H}$ NMR (400 MHz, DMSO- $d_{6}$ ) $\delta: 10.73$ (s, 1H), 8.13 (d, $J=8.6 \mathrm{~Hz}, 2 \mathrm{H}), 8.08$ (d, $J=15.4$ $\mathrm{Hz}, 1 \mathrm{H}), 7.96$ (d, $J=7.5 \mathrm{~Hz}, 1 \mathrm{H}), 7.87$ (t, $J=7.7 \mathrm{~Hz}, 1 \mathrm{H})$, 7.81 (d, $J=8.6 \mathrm{~Hz}, 2 \mathrm{H}), 7.69$ (d, $J=7.8 \mathrm{~Hz}, 1 \mathrm{H}), 7.64$ (d, $J=15.4 \mathrm{~Hz}, 1 \mathrm{H}), 4.33(\mathrm{~s}, 2 \mathrm{H}) ;{ }^{13} \mathrm{C} \mathrm{NMR}(101 \mathrm{MHz}$, DMSO- $\left.d_{6}\right) \delta: 187.59,165.27,154.27,143.18,141.66$, 
$140.62,140.46,132.31,130.08,128.96,126.29,124.33$, 118.88, 43.62. HRMS (ESI) calcd for $\mathrm{C}_{16} \mathrm{H}_{13} \mathrm{BrClN}_{2} \mathrm{O}_{2}$ $[\mathrm{M}+\mathrm{H}]^{+}: 378.9849$, found 378.9850 .

3.2.13(E)-3-氯- $N$ - $\{4$-[3-(2-吡啶基)丙烯酰基]苯基 $\}$ 丙酰胺 $(5 e)$ 的合成

操作同 5a 的合成, 产物为黄色固体, 收率为 $78 \%$. m.p. $74 \sim 75{ }^{\circ} \mathrm{C} ;{ }^{1} \mathrm{H}$ NMR $\left(400 \mathrm{MHz}, \mathrm{CDCl}_{3}\right) \delta: 8.62(\mathrm{~d}$, $J=3.9 \mathrm{~Hz}, 1 \mathrm{H}), 8.15(\mathrm{~s}, 1 \mathrm{H}), 8.02(\mathrm{dd}, J=11.9,10.7 \mathrm{~Hz}$, $3 \mathrm{H}), 7.74 \sim 7.59(\mathrm{~m}, 4 \mathrm{H}), 7.41(\mathrm{~d}, J=7.7 \mathrm{~Hz}, 1 \mathrm{H}), 7.28 \sim$ $7.21(\mathrm{~m}, 1 \mathrm{H}), 3.81(\mathrm{t}, J=6.4 \mathrm{~Hz}, 2 \mathrm{H}), 2.81(\mathrm{t}, J=6.4 \mathrm{~Hz}$, $2 \mathrm{H}) ;{ }^{13} \mathrm{C}$ NMR $\left(101 \mathrm{MHz}, \mathrm{CDCl}_{3}\right) \delta: 189.08,168.25$, $153.13,150.10,142.56,142.15,137.07,133.63,130.25$, $125.53,125.39,124.54,119.26,40.49,39.64$. HRMS (ESI) calcd for $\mathrm{C}_{17} \mathrm{H}_{16} \mathrm{ClN}_{2} \mathrm{O}_{2}[\mathrm{M}+\mathrm{H}]^{+}$: 315.0900 , found 315.0898 .

3.2.14 (E)-4-氯- $N$ - $\{4$-[3-(2-吡啶基)丙烯酰基]苯基 $\}$ 丁酰胺 $(\mathbf{5 f})$ 的合成

操作同 $\mathbf{5 a}$ 的合成, 产物为黄色固体, 收率为 $78 \%$. m.p. $155 \sim 156{ }^{\circ} \mathrm{C} ;{ }^{1} \mathrm{H}$ NMR (400 MHz, $\mathrm{CDCl}_{3}$ ) $\delta: 8.69$ (d, $J=4.1 \mathrm{~Hz}, 1 \mathrm{H}), 8.11(\mathrm{t}, J=12.4 \mathrm{~Hz}, 3 \mathrm{H}), 7.99(\mathrm{~s}, 1 \mathrm{H})$, $7.82 \sim 7.66(\mathrm{~m}, 4 \mathrm{H}), 7.48(\mathrm{~d}, J=7.7 \mathrm{~Hz}, 1 \mathrm{H}), 7.31(\mathrm{dd}, J=$ $7.1,5.1 \mathrm{~Hz}, 1 \mathrm{H}), 3.66$ (t, $J=6.1 \mathrm{~Hz}, 2 \mathrm{H}), 2.62$ (t, $J=7.0$ $\mathrm{Hz}, 2 \mathrm{H}), 2.25 \sim 2.15(\mathrm{~m}, 2 \mathrm{H}) ;{ }^{13} \mathrm{C}$ NMR $(101 \mathrm{MHz}$, $\left.\mathrm{CDCl}_{3}\right) \delta: 188.99,170.44,153.15,150.10,142.48,142.40$, 137.04, 133.44, 130.27, 125.50, 125.40, 124.49, 119.04, 44.37, 34.19, 27.70. HRMS (ESI) calcd for $\mathrm{C}_{18} \mathrm{H}_{18} \mathrm{ClN}_{2} \mathrm{O}_{2}$ $[\mathrm{M}+\mathrm{H}]^{+}: 329.1057$, found 329.1056 .

3.2.15( $(E)-5$-氯- $N$ - $\{4$-[3-(2-吡啶基)丙烯酰基]苯基 $\}$ 戊酰胺 $(5 g)$ 的合成

操作同 $\mathbf{5 a}$ 的合成, 产物为黄色固体, 收率为 $72 \%$. m.p. $67 \sim 68{ }^{\circ} \mathrm{C} ;{ }^{1} \mathrm{H}$ NMR $\left(400 \mathrm{MHz}, \mathrm{CDCl}_{3}\right) \delta: 8.68(\mathrm{~d}$, $J=4.0 \mathrm{~Hz}, 1 \mathrm{H}), 8.17 \sim 7.91(\mathrm{~m}, 4 \mathrm{H}), 7.82 \sim 7.62(\mathrm{~m}, 4 \mathrm{H})$, $7.47(\mathrm{~d}, J=7.7 \mathrm{~Hz}, 1 \mathrm{H}), 7.30(\mathrm{dd}, J=7.0,5.1 \mathrm{~Hz}, 1 \mathrm{H})$, 3.56 (d, $J=5.3 \mathrm{~Hz}, 2 \mathrm{H}), 2.44$ (t, $J=6.6 \mathrm{~Hz}, 2 \mathrm{H}), 1.86$ (s, $4 \mathrm{H}) ;{ }^{13} \mathrm{C}$ NMR $\left(101 \mathrm{MHz}, \mathrm{CDCl}_{3}\right) \delta: 188.00,170.13$, $152.14,149.10,141.48,136.01,132.33,129.24,124.46$, 124.36, 123.48, 118.01, 43.54, 35.66, 30.81, 21.60. HRMS (ESI) calcd for $\mathrm{C}_{19} \mathrm{H}_{20} \mathrm{ClN}_{2} \mathrm{O}_{2}[\mathrm{M}+\mathrm{H}]^{+}: 343.1213$, found 343.1216.

3.2.16(E)-2-氯- $N$ - $\{2$-[3-(2-吡啶基)丙烯酰基]苯基 $\}$ 乙酰胺 $(5 h)$ 的合成

操作同 $\mathbf{5 a}$ 的合成, 产物为黄色固体, 收率为 $79 \%$. m.p. $157 \sim 158{ }^{\circ} \mathrm{C} ;{ }^{1} \mathrm{H}$ NMR (400 MHz, $\left.\mathrm{CDCl}_{3}\right) \delta: 12.43$ (s, 1H), 8.72 (dd, $J=12.7,6.3 \mathrm{~Hz}, 2 \mathrm{H}), 8.18$ (dd, $J=20.0$, $11.3 \mathrm{~Hz}, 2 \mathrm{H}), 7.84 \sim 7.73(\mathrm{~m}, 2 \mathrm{H}), 7.62(\mathrm{t}, J=7.9 \mathrm{~Hz}, 1 \mathrm{H})$, $7.48(\mathrm{~d}, J=7.7 \mathrm{~Hz}, 1 \mathrm{H}), 7.33(\mathrm{dd}, J=7.2,4.9 \mathrm{~Hz}, 1 \mathrm{H})$, $7.29 \sim 7.21(\mathrm{~m}, 2 \mathrm{H}), 4.23$ (s, 2H); ${ }^{13} \mathrm{C}$ NMR (101 MHz, $\left.\mathrm{CDCl}_{3}\right) \delta: 193.32,165.66,152.76,150.24,143.62,140.01$, $137.05,134.92,131.24,126.29,125.85,124.73,123.87$, 123.52, 121.09, 43.30. HRMS (ESI) calcd for $\mathrm{C}_{16} \mathrm{H}_{14} \mathrm{Cl}-$ $\mathrm{N}_{2} \mathrm{O}_{2}[\mathrm{M}+\mathrm{H}]^{+}: 301.0744$, found 301.0746 .

3.2.17( $(E)-2$ - 氯- $N$ - $\{3$-[3-(2-吡啶基)丙烯酰基]苯基 $\}$ 乙酰胺 $(5 i)$ 的合成

操作同 $5 \mathrm{a}$ 的合成, 产物为黄色固体, 收率为 $65 \%$. m.p. $117 \sim 118{ }^{\circ} \mathrm{C} ;{ }^{1} \mathrm{H}$ NMR (400 MHz, $\left.\mathrm{CDCl}_{3}\right) \delta: 8.63$ (d, $J=3.5 \mathrm{~Hz}, 1 \mathrm{H}), 8.36$ (s, 1H), 7.99 (t, $J=15.4 \mathrm{~Hz}, 3 \mathrm{H})$, $7.83(\mathrm{~d}, J=7.5 \mathrm{~Hz}, 1 \mathrm{H}), 7.70(\mathrm{dd}, J=20.0,11.5 \mathrm{~Hz}, 2 \mathrm{H})$, $7.44(\mathrm{dd}, J=13.8,7.4 \mathrm{~Hz}, 2 \mathrm{H}), 7.28 \sim 7.21(\mathrm{~m}, 1 \mathrm{H}), 4.16$ (s, 2H); ${ }^{13} \mathrm{C}$ NMR $\left(101 \mathrm{MHz}, \mathrm{CDCl}_{3}\right) \delta: 189.78,164.21$, $153.01,150.19,143.25,138.64,137.32,137.01,129.64$, $125.58,125.42,125.27,124.73,124.61,120.04,42.88$. HRMS (ESI) calcd for $\mathrm{C}_{16} \mathrm{H}_{14} \mathrm{ClN}_{2} \mathrm{O}_{2}[\mathrm{M}+\mathrm{H}]^{+}: 301.0744$, found 301.0747 .

3.2.18 (E)- $N$ - \{4-[3-(2-吡啶基)丙烯酰基]苯基 $\} 乙$ 酰 胺 $(\mathbf{5 j})$ 的合成

操作同 $5 \mathbf{a}$ 的合成, 产物为淡黄色固体, 收率为 84\%. m.p. $183 \sim 184{ }^{\circ} \mathrm{C} ;{ }^{1} \mathrm{H}$ NMR (400 MHz, $\left.\mathrm{CDCl}_{3}\right) \delta$ : 8.69 (s, 1H), 8.10 (t, $J=11.2 \mathrm{~Hz}, 3 \mathrm{H}), 7.98$ (s, 1H), 7.73 (dd, $J=33.0,11.1 \mathrm{~Hz}, 4 \mathrm{H}), 7.48$ (d, $J=7.3 \mathrm{~Hz}, 1 \mathrm{H}), 7.30$ (d, $J=6.0 \mathrm{~Hz}, 1 \mathrm{H}), 2.22$ (s, 3H); ${ }^{13} \mathrm{C}$ NMR $(101 \mathrm{MHz}$, $\left.\mathrm{CDCl}_{3}\right) \delta$ : 189.02, 168.74, 153.22, 150.14, 142.54, 142.49, $136.99,133.40,130.24,125.45,125.40,124.46,118.98$, 24.77. HRMS (ESI) calcd for $\mathrm{C}_{16} \mathrm{H}_{15} \mathrm{~N}_{2} \mathrm{O}_{2}[\mathrm{M}+\mathrm{H}]^{+}$: 267.1134, found 267.1132 .

\subsubsection{9 (E)-2-溴- $N$-\{4-[3-(2-吡啶基)丙烯酰基]苯基 $\}$} 乙酰胺 $(5 k)$ 的合成

操作同 $\mathbf{5 a}$ 的合成, 产物为黄色固体, 收率为 $84 \%$. m.p. $185 \sim 186{ }^{\circ} \mathrm{C}$; ${ }^{1} \mathrm{H}$ NMR (400 MHz, DMSO- $d_{6}$ ) $\delta$ : $10.80(\mathrm{~s}, 1 \mathrm{H}), 8.70(\mathrm{~d}, J=3.6 \mathrm{~Hz}, 1 \mathrm{H}), 8.16(\mathrm{t}, J=11.4 \mathrm{~Hz}$, 4H), 7.92 (d, $J=3.2 \mathrm{~Hz}, 3 \mathrm{H}), 7.81$ (d, $J=8.2 \mathrm{~Hz}, 2 \mathrm{H}), 7.72$ (d, $J=15.4 \mathrm{~Hz}, 1 \mathrm{H}), 7.45$ (d, $J=4.1 \mathrm{~Hz}, 1 \mathrm{H}), 4.11(\mathrm{~s}, 2 \mathrm{H})$; ${ }^{13} \mathrm{C}$ NMR (101 MHz, DMSO- $\left.d_{6}\right) \quad \delta$ : 187.79, 165.42, $152.77,149.93,143.14,142.49,137.26,132.53,129.98$, 125.06, 124.92, 124.78, 118.78, 30.29. HRMS (ESI) calcd for $\mathrm{C}_{16} \mathrm{H}_{14} \mathrm{BrN}_{2} \mathrm{O}_{2}[\mathrm{M}+\mathrm{H}]^{+}:$345.0239, found 345.0239.

3.2.20 (E)-2-氯- $N$ - \{4-[3-(吡啶-3-基)丙烯酰基]苯基 $\}$ 乙酰胺 $(51)$ 的合成

操作同 $5 \mathbf{a}$ 的合成, 产物为黄色固体, 收率为 $85 \%$. m.p. $242 \sim 243{ }^{\circ} \mathrm{C} ;{ }^{1} \mathrm{H}$ NMR (400 MHz, DMSO- $\left.d_{6}\right) \delta$ : $10.71(\mathrm{~s}, 1 \mathrm{H}), 9.03(\mathrm{~d}, J=1.3 \mathrm{~Hz}, 1 \mathrm{H}), 8.62(\mathrm{~d}, J=3.8 \mathrm{~Hz}$, 
$1 \mathrm{H}), 8.36(\mathrm{~d}, J=8.0 \mathrm{~Hz}, 1 \mathrm{H}), 8.21(\mathrm{~d}, J=8.7 \mathrm{~Hz}, 2 \mathrm{H}), 8.10$ (d, $J=15.7 \mathrm{~Hz}, 1 \mathrm{H}), 7.78$ (dd, $J=14.9,12.3 \mathrm{~Hz}, 3 \mathrm{H}), 7.51$ (dd, $J=7.9,4.8 \mathrm{~Hz}, 1 \mathrm{H}), 4.34(\mathrm{~s}, 2 \mathrm{H}) ;{ }^{13} \mathrm{C}$ NMR $(101$ $\mathrm{MHz}$, DMSO- $\left.d_{6}\right) \quad \delta: 187.32,165.25,150.92,150.30$, $143.02,140.06,135.06,132.53,130.57,130.09,123.90$, 123.78, 118.74, 43.61. HRMS (ESI) calcd for $\mathrm{C}_{16} \mathrm{H}_{14} \mathrm{Cl}-$ $\mathrm{N}_{2} \mathrm{O}_{2}[\mathrm{M}+\mathrm{H}]^{+}: 301.0744$, found 301.0742 .

3.2.21 (E)-2-氯- $\mathrm{N}$-\{4-[3-(吡啶基)丙烯酰基]苯基 $\} 乙$ 酰胺 $(\mathbf{5 m})$ 的合成

操作同 $\mathbf{5 a}$ 的合成, 产物为黄色固体, 收率为 $87 \%$. m.p. $224 \sim 225{ }^{\circ} \mathrm{C} ;{ }^{1} \mathrm{H}$ NMR (400 MHz, DMSO- $\left.d_{6}\right) \delta$ : $10.76(\mathrm{~s}, 1 \mathrm{H}), 10.76(\mathrm{~s}, 1 \mathrm{H}), 8.67(\mathrm{~d}, J=4.9 \mathrm{~Hz}, 2 \mathrm{H}), 8.20$ (d, $J=8.6 \mathrm{~Hz}, 2 \mathrm{H}), 8.16$ (d, $J=15.9 \mathrm{~Hz}, 1 \mathrm{H}), 7.84$ (d, $J=$ $5.0 \mathrm{~Hz}, 2 \mathrm{H}), 7.80$ (d, $J=8.5 \mathrm{~Hz}, 2 \mathrm{H}), 7.66(\mathrm{~d}, J=15.7 \mathrm{~Hz}$, $1 \mathrm{H}), 4.33$ (s, 2H); ${ }^{13} \mathrm{C}$ NMR (101 MHz, DMSO- $\left.d_{6}\right) \delta$ : 187.46, 165.28, 150.31, 143.20, 141.88, 140.54, 132.31, 130.21, 126.27, 122.48, 118.76, 43.61. HRMS (ESI) calcd for $\mathrm{C}_{16} \mathrm{H}_{14} \mathrm{ClN}_{2} \mathrm{O}_{2}[\mathrm{M}+\mathrm{H}]^{+}: 301.0744$, found 301.0743 .

\section{3 生物活性评价}

\subsection{1 实验仪器与材料}

超纯水仪(Thermo); 生物安全柜 HR40-HA2(青岛海 耳公司); 恒温摇床 ZMY-1102C(河南超继仪器设备公 司); 微量可调节加样器(德国 Eeppendorf); 自动立式压 力蒸汽灭菌器 MJ-78A 型(河南超继仪器设备公司); 96 孔细胞培养板(NEST); $-20{ }^{\circ} \mathrm{C}$ 冰箱 BCD-232 型(伊莱 克斯); $-80{ }^{\circ} \mathrm{C}$ 超低温冰箱(德国 Thermo Scientific Inc.); 米勒-海顿(MHA)琼脂培养基, 米勒-海顿(MHB)肉汤培 养基, 卢里亚一贝尔塔尼(LB)培养基均产自北京奥博星 生物技术有限责任公司.

\subsection{2 微量肉汤法测定最小抑菌浓度}

选择革兰氏阳性的金黄色葡萄球菌(ATCC 29213) 革兰氏阴性的大肠埃希菌(ATCC 25922)为评价对象, 实 验操作如下 ${ }^{[2]}$ : 参照美国 CLSI M07-A9 标准中规定的 微量肉汤稀释法测定化合物 $5 \mathrm{a} \sim 5 \mathrm{~m}$ 的最小抑菌浓度 (MIC)值, 分别以空白肉汤和待测菌液为阴性和阳性对 照组. 使细菌于 $37{ }^{\circ} \mathrm{C} 250 \mathrm{rpm}$ 恒温摇床中培养 $6 \mathrm{~h}$ 使 其生长至 $1 \times 10^{8} \mathrm{CFU} / \mathrm{mL}$. 用合适的培养基(ATCC 29213, MRSA 为 MHB; ATCC 25922 为 LB) 稀释菌液至 $10^{5} \mathrm{CFU} / \mathrm{mL}$. 所有的待测药物 $(\mathbf{5 a} \sim \mathbf{5 m})$ 用无菌水溶解 为合适浓度的待测储备液于 $-80{ }^{\circ} \mathrm{C}$ 冰箱内储存备用. 实验开始, 先将 $200 \mu \mathrm{L}$ 化合物待测储备液加入 96 孔板 中, 用合适的培养基对其进行梯度稀释. 最后, 将 100 $\mu \mathrm{L}$ 稀释好的 $10^{5} \mathrm{CFU} / \mathrm{mL}$ 菌液加入已经梯度稀释好的含 药 96 孔板中. $37{ }^{\circ} \mathrm{C}$ 培养 16 20 h. 读取最小抑菌浓度 (MIC)结果, 以肉眼观察所能完全抑制细菌生长的浓度
判定为该待测化合物的 MIC.

\subsection{3 红细胞溶血率测定}

采用新鲜脱纤维无菌羊血分离得到的红细胞(Red blood cells, RBCs), 将无菌羊血用磷酸缓冲盐溶液(PBS) 重悬后配置为红细胞悬液 (5\%), 向 96 孔板中加入 150 $\mu \mathrm{L}$ 的 $5 \%$ 的红细胞悬液, 随后加入 $50 \mu \mathrm{L}$ 稀释好的待测 化合物, 阴性对照和阳性对照分别为 $50 \mu \mathrm{L}$ PBS 溶液和 $0.1 \%$ 的曲拉通 X-100. 将 96 孔板放入 $37{ }^{\circ} \mathrm{C}$ 恒温培养箱 䁔育 $1 \mathrm{~h}$. 睬育结束后, 将 96 孔板 $3500 \mathrm{r} / \mathrm{min}$ 离心 $5 \mathrm{~min}$. 吸出 $100 \mu \mathrm{L}$ 上清至另一干净的 96 孔板中, 于酶标仪 540 $\mathrm{nm}$ 波长处测其 $\mathrm{OD}$ 值. 红细胞溶血率 $(\%)=(A-$ $\left.A_{0}\right) /\left(A_{\text {total }}-A_{0}\right) \times 100 \% . A$ 为待测药物的吸光度, $A_{0}$ 为阴 性对照组吸光度, $\mathrm{A}_{\text {total }}$ 为用曲拉通 X-100 处理后全溶血. 的阳性对照组的吸光度 ${ }^{[23]}$.

辅助材料(Supporting Information) 化合物 $\mathbf{3 a} \sim 3 \mathbf{h}$ 和 5a $\sim 5 \mathrm{~m}$ 的核磁氢谱和核磁碳谱. 这些材料可以免费从 本刊网站(http://sioc-journal.cn/)上下载.

\section{References}

[1] Jevons, M. P. Br. Med.J. 1961, 1, 124.

[2] Hamilton, G. L. A. Lab. Med. 2010, 41, 329.

[3] Deurenberg, R. H.; Nulens, E.; Valvatne, H.; Sebastian, S.; Driessen, C.; Craeghs, J.; De Brauwer, E.; Heising, B.; Kraat, Y. J.; Riebe, J.; Stals, F. S.; Trienekens, T. A.; Scheres, J.; Friedrich, A. W.; van Tiel, F. H.; Beisser, P. S.; Stobberingh, E. E. Emerging Infect. Dis. 2009, 15, 727.

[4] Kallen, A. J.; Mu, Y.; Bulens, S.; Reingold, A.; Petit, S.; Gershman, K.; Ray, S. M.; Harrison, L. H.; Lynfield, R.; Dumyati, G. JAMA, J. Am. Med. Assoc. 2010, 304, 641.

[5] Fridkin, S. K.; Hageman, J. C.; Morrison, M.; Sanza, L. T.; Como-Sabetti, K.; Jernigan, J. A.; Harriman, K.; Harrison, L. H.; Lynfield, R.; Farley, M. M. N. Engl. J. Med. 2005, 352, 1436.

[6] Cardo, D.; Horan, T.; Andrus, M.; Dembinski, M.; Edwards, J.; Peavy, G.; Tolson, J.; Wagner, D. Am. J. Infect. Control. 2004, 32, 470.

[7] Fridkin, S. K.; Yokoe, D. S.; Whitney, C. G.; Onderdonk, A.; Hooper, D. C. J. Clin. Microbiol. 1998, 36, 965.

[8] Rybak, M. J.; Abate, B. J.; Kang, S. L.; Ruffing, M. J.; Lerner, S. A.; Drusano, G. L. Antimicrob. Agents Chemother. 1999, 43, 1549.

[9] Nowakowska, Z. Eur. J. Med. Chem. 2007, 42, 125.

[10] Magiorakos, A. P.; Srinivasan, A.; Carey, R. B.; Carmeli, Y.; Falagas, M. E.; Giske, C. G.; Harbarth, S.; Hindler, J. F.; Kahlmeter, G.; Olsson-Liljequist, B.; Paterson, D. L.; Rice, L. B.; Stelling, J.; Struelens, M. J.; Vatopoulos, A.; Weber, J. T.; Monnet, D. L. Clin. Microbiol. Infect. 2012, 18, 268.

[11] Kluytmans, J.; Van Belkum, A.; Verbrugh, H. Clin. Microbiol. Rev. 1997, 10, 505.

[12] Patel, R. V.; Patel, P. K.; Kumari, P.; Rajani, D. P.; Chikhalia, K. H. Eur. J. Med. Chem. 2012, 53, 41.

[13] Chen, Z.-H.; Zheng, C.-J.; Sun, L.-P.; Piao, H.-R. Eur. J. Med. Chem. 2010, 45, 5739.

[14] Klevens, R. M.; Morrison, M. A.; Nadle, J.; Petit, S.; Gershman, K.; Ray, S.; Harrison, L. H.; Lynfield, R.; Dumyati, G.; Townes, J. M. 
JAMA, J. Am. Med. Assoc. 2007, 298, 1763.

[15] Zhang, Q.-R.; Xue, D.-Q.; He, P.; Shao, K.-P.; Chen, P.-J.; Gu, Y.-F.; Ren, J.-L.; Shan, L.-H.; Liu, H.-M. Bioorg. Med. Chem. Lett. 2014, 24, 1236.

[16] Chen, P.-J.; Yang, A.; Gu, Y.-F.; Zhang, X.-S.; Shao, K.-P.; Xue, D.-Q.; He, P.; Jiang, T.-F.; Zhang, Q.-R.; Liu, H.-M. Bioorg. Med. Chem. Lett. 2014, 24, 2741.

[17] Nielsen, S. F.; Larsen, M.; Boesen, T.; Schønning, K.; Kromann, H. J. Med. Chem. 2005, 48, 2667.

[18] Stringer, J. R.; Bowman, M. D.; Weisblum, B.; Blackwell, H. E. ACS Comb. Sci. 2011, 13, 175.

[19] Joshi, A. S.; Li, X. C.; Nimrod, A. C.; ElSohly, H. N.; Walker, L. A.; Clark, A. M. Planta Med. 2001, 67, 186.

[20] Fu, D.-J.; Zhang, S.-Y.; Liu, Y.-C.; Yue, X.-X.; Liu, J.-J.; Song, J.;
Zhao, R.-H.; Li, F.; Sun, H.-H.; Zhang, Y.-B.; Liu, H.-M. Med. Chem. Commun. 2016, 7, 1664

[21] Fu, D.-J.; Zhang, S.-Y.; Liu, Y.-C.; Zhang, L.; Liu, J.-J.; Song, J.; Zhao, R.-H.; Li, F.; Sun, H.-H.; Liu, H.-M.; Zhang, Y.-B. Bioorg. Med. Chem. Lett. 2016, 26, 3918.

[22] Ling, L. L.; Schneider, T.; Peoples, A. J.; Spoering, A. L.; Engels, I.; Conlon, B. P.; Mueller, A.; Schaberle, T. F.; Hughes, D. E.; Epstein, S.; Jones, M.; Lazarides, L.; Steadman, V. A.; Cohen, D. R.; Felix, C. R.; Fetterman, K. A.; Millett, W. P.; Nitti, A. G.; Zullo, A. M.; Chen, C.; Lewis, K. Nature 2015, 517, 455.

[23] Ghosh, C.; Manjunath, G. B.; Akkapeddi, P.; Yarlagadda, V.; Hoque, J.; Uppu, D. S.; Konai, M. M.; Haldar, J. J. Med. Chem. 2014, 57, 1428.

(Li, L.; Fan, Y.) 